\title{
Introduction to the special section: Recent advancement on integrated carbonate reservoirs prediction with complex secondary storage space
}

\author{
(C) China University of Petroleum (Beijing) and Springer-Verlag Berlin Heidelberg 2011
}

Carbonate reservoirs in Western China show tremendous exploration and development potential. These have become the key area for oil and gas reserves addition and sustainable resource development for China. Compared to conventional carbonate reservoirs, prediction of this kind of reservoir predominated by secondary storage is much more difficult and presents significant challenges. Seismic detection of dissolution pores and fractures always requires a high standard of technology, which puts further serious challenges on exploration and creates many technological issues for field development plans. The key geophysical problems are tackled based on a detailed analysis of exploration issues for such complex carbonate reservoirs. Subsequently, a set of techniques for complex carbonate reservoir prediction was established, including pre-stack amplitude-preserved processing, new rock physics models, pre-stack AVO inversion, seismic multi-attribute optimization and integrated prediction, fracture detection using pre-stack data with limited azimuthal distribution and fluid identification based on prestack inversion. Additionally, to solve some special problems, diffraction wave field separation and imaging, nonstationary deconvolution algorithms are also developed and employed. Moreover, application of this set of techniques to field data has proven to be effective and feasible and drilling results show good agreement with our research. Nevertheless, the field is still in its infancy.

The basis for carbonate reservoir prediction is rock physics research which can build a bridge between seismic information and rock physical properties, and it is the premise for pre-stack inversion, reservoir characterization and quantitative reservoir prediction. Paper 1 discusses a rock physics study of complex carbonate reservoirs in the Tarim Basin. It mainly centers on the effects of the secondary storage spaces on the rock's elastic properties, seismic wave velocity, and reservoir permeability, all of which are important for quantitative reservoir characterization. Amplitude-preserved seismic processing is the foundation for pre-stack inversion, and is also the key for reservoir imaging and prediction. Paper 2 introduces an integrated processing workflow for amplitude preservation that is suitable for data from the Tarim Basin. Complex carbonate reservoir prediction requires the study of reservoir heterogeneity and anisotropy, because the former is caused by dissolved pores and caves, and the latter is caused by fractures. Paper 3 uses the AVO inversion method to study heterogeneity in carbonate reservoirs. Considering the fact that the seismic data in the Tarim Basin are mostly narrow azimuth, a P-wave fracture prediction algorithm using pre-stack data with limited azimuth is proposed in paper 4 . This technology enables fracture parameters to be obtained from seismic data with narrow azimuth and relatively low signal-to-noise ratio, and facilitates the detection of fractured reservoirs. Pre-stack fracture prediction enables subsequent fracture fluid prediction and fractured reservoir modeling. The Discrete Fracture Network (DFN) modeling is one of the important step for fractured reservoir characterization. This method is proposed in paper 5 to build an equivalent model of a fractured reservoir for further reservoir simulation. Forward and inverse seismic modeling is performed on this model to establish the relationship between seismic response characteristics and fracture parameters. Complex carbonate reservoirs are not only difficult to predict, but also have some specific challenges for formation evaluation. Paper 6 describes some new techniques in evaluating carbonate reservoir formation. Finally, 3D seismic attribute optimization technology is discussed in paper 7. Comprehensive analysis and optimization of seismic attributes is the key for pre- and post-stack integration, and can make full use of the seismic information to improve the precision of reservoir prediction. Based on previous research achievements, Lab for Integration of Geology and Geophysics (LIGG) is currently undertaking in-depth research in some more advanced techniques, including carbonate rock physics models dealing dispersion and attenuation, the quantitative inversion of pore types, azimuth gather extraction algorithms for AVO inversion based on reverse time migration, fluid detection using pre-stack inversion, Q migration and non-stationary deconvolution to enhance resolution, diffraction wave field separation technology for better imaging of dissolved pores and caves. LIGG group will continue to work on complicated carbonate reservoir research and make due contributions to China's oil and gas exploration.

Paper 1: The influence of pore structure on P- \& S-wave velocities in complex carbonate reservoirs with secondary storage space

Paper 2: The application of amplitude-preserved processing and migration for carbonate reservoir prediction in the Tarim Basin, China

Paper 3: Pre-stack inversion for caved carbonate reservoir prediction: A case study from Tarim Basin, China

Paper 4: P-wave fracture prediction algorithm using prestack data with limited azimuth distribution: A case study in the TZ45 area, Tarim Basin, China 
Paper 5: Fractured reservoir modeling by discrete fracture network and seismic modeling in the Tarim Basin, China

Paper 6: A new method of formation evaluation for fractured and caved carbonate reservoirs: A case study from the Lundong area, Tarim Basin, China

Paper 7: Seismic attributes and integrated prediction of fractured and caved carbonate reservoirs in the Tarim Basin, China

The Lab for Integration of Geology and Geophysics (LIGG) (Web: www.liggr.org) was established in September 2007 at the China University of Petroleum (Beijing), sponsored by BGP Ltd., Tarim Oilfield Co., Liaohe Oilfield Co., and Department of Science and Technology Management, CNPC. It has close collaboration with the State Key Laboratory of Petroleum Resource and Prospecting and CNPC Geophysical Key Lab. LIGG research group consists of 29 main research members including professor, associate professor, lecturer, $\mathrm{PhD}$ students and postgraduates.

LIGG is aimed at researching and developing advanced technology for petroleum exploration, production and reservoir management for the best economic return-the highest oil recovery, and the lowest cost. Another goal of LIGG is to educate and train world-class technical leaders for the petroleum industry. They are focusing on practical issues that oilfields are facing. Meanwhile they take all opportunities to work on theoretical problems that will further geophysical science. It is believed in LIGG that elastic information is very important for complex reservoir characterization and pre-stack data have a lot of potentials for providing that information. Currently, LIGG is mostly focusing on the following research topics:

- Amplitude-preserved processing and migration for common reflection point (CRP) gathering, especially gathering algorithms from Kirchhoff migration, wave equation migration and reverse time migration (RTM).

- Pre-stack inversion algorithm development including pre-stack PP-wave, PS-wave, and joint PP and PS inversion algorithms for both heterogeneity and anisotropy reservoir, e.g. fracture detection algorithm with limited azimuthal distribution.

- Rock physics model studies and application focusing on both $\mathrm{P}$ and $\mathrm{S}$ wave velocity prediction by considering fluid saturation and pore structure. This research is an important bridge that links reservoir property to seismic data via prestack inversion.

- A lot of fundamental research is carried out in LIGG for 3D3C VSP which is an important tool for reservoir geophysics. These researches focus on vector wavefield separation, velocity model building, VSP stacking and migration.

Equipments

- 800 cores (100 node IBM dual CPU 4-core) super computer and associated high-speed and high-performance storage (62T);

-Additional high-performance storage (114T);
-23 Linux/Unix workstations and associated storage (100T);

$\bullet 30$ high-end linux terminal computers;

-22 high-end PCs;

-A set of high-resolution plotters;

- A set of high-end color and BW laser printers;

$\bullet 1 \mathrm{~Gb} / \mathrm{s}$ office network;

-Commercial geological and geophysical software;

-Advanced E\&P software developed by LIGG.

Sam Zandong Sun is a professor and director of Lab for Integration of Geology and Geophysics (LIGG) at China University of Petroleum (Beijing), who is also the senoir technical advisor for BGP Ltd. Prior to joining China University of Petroleum, Sam held a variety of positions with functions of exploration, production, and research as a senoir research specialist with ExxonMobil Upstream Research, ExxonMobil Production Company, and Imperial Oil (ESSO Canada) for nearly 14 years. He also worked with Schlumberger, former Mobil Canada, and former Canadian Hunter Exploration Ltd.

Professor Sun worked with CNPC headquarter research for about three years after graduating from his B.Sc. (1984) in geology from Jianghan Petroleum Institute. He earned both of his M.Sc. (1993) and Ph.D. (1999) in geophysics from University of Calgary, Canada. Sam has been focusing on reservoir monitoring, characterization, and prediction utilizing $\mathrm{P}$-wave and 3D3C pre-stack data by integrating rock physics with amplitude processing including amplitude preserved migration and inversion for rservoir prediction and fluid mapping. Elastic information is very critical for reservoir and fluid prediction.

To compromise high acquisition cost, Sam strongly believes that pre-stack PP wave data should be worked harder and employed more often to extract elastic information for reservoir and fluid mapping. For that he leads the group to focus on integration of rock physics, amplitude processing, and inversion algorithms and new technology development to improve these integrations.

Recently, Sam has had a strong interest in unconventional resource development and published the book "Uncoventional Oil \& Gas Exploration and Development". He also worked on 3C3D VSP technology development for a long time and published the book "3C3D VSP: Principle and Applications".

Sam has been very active in serving the society. He is a member of SEG Global Affairs, Research, D\&P, and Reserve committees. Also, he serves as a member of SEG Beijing task force and is a member of SEG China advisory committee. He is an active member of SEG, AAPG, EAGE and a member of APPEGA. He served as chairman of 2011 SEG D\&P Forum. Sam is elected by SEG as an Honorary Lecturer for South and East Asia in 2012. He was listed as a member of USA National Register's "WHO IS WHO” in Executives and Professionals during 2005-2006 (Registered in the congress library of USA). 